\title{
Ambiguity of Propranolol Side Effects in 100 Patients Treated for Infantile Hemangioma
}

\author{
Danielle $\mathrm{H}^{1,2^{*}}$, Dor $\mathrm{L}^{2}$ and Avraham $\mathrm{L}^{3}$ \\ ${ }^{1}$ Rappaport Faculty of Medicine, Technion-Israel Institute of Technology, Haifa, Israel \\ ${ }^{2}$ Chaim Sheba Medical Center, Tel HaShomer, Ramat Gan, Israel \\ ${ }^{3}$ Pediatric Cardiology Institute, Rambam Health Care Campus, Haifa, Israel
}

*Corresponding author: Danielle H, Chaim Sheba Medical Center, Tel HaShomer, Ramat Gan, Israel, Tel: +972 50 2262 005 ; E-mail: dani2612@gmail.com

Received date: February 16, 2016; Accepted date: January 23, 2018; Published date: January 27, 2018

Citation: Danielle H, Dor L, Avraham L (2018) Ambiguity of Propranolol Side Effects in 100 Patients Treated for Infantile Hemangioma. Arch Med Vol No:10 Iss No:1:4

Copyright: @ 2018 Danielle $\mathrm{H}$, et al. This is an open-access article distributed under the terms of the Creative Commons Attribution License, which permits unrestricted use, distribution, and reproduction in any medium, provided the original author and source are credited.

\begin{abstract}
Background: Infantile hemangiomas are the most common tumor of infancy. Propranolol hydrochloride has been prescribed for decades in the pediatric population for a variety of disorders. Since 2008 propranolol has replaced corticosteroids to become the first line of treatment for infantile hemangioma due to its high effectiveness and low rate of side effects. Of the side effects reported, the most serious yet rare side effects include: hypoglycemia, bronchial hyper-responsiveness, hypotension and bradycardia.
\end{abstract}

Objective: To describe the effects and side effects of propranolol treatment among 100 pediatric patients.

Conclusion: Propranolol has a long history of safe and effective use in infants and children, and was well tolerated in our study. Understanding and recognition of deleterious adverse effects is critical for physicians and caregivers. Outpatient treatment is possible if parents follow strict guidelines. Educating parents about mild and transient symptoms could prevent unnecessary concern.

Keywords: Hemangioma; Propranolol; Side effects; Bradycardia

\section{Introduction}

Hemangiomas are benign vascular tumors that are a quite common finding among neonates and infants ( $4 \%$ to $10 \%$ of all Caucasian infants) [1]. Infantile hemangiomas typically present a few weeks after birth and occur more frequently among females [2]. The characteristic course of infantile hemangiomas begins with a rapid growth phase in the first months of life, followed by a plateau and then an involution phase, a process which can last several years [3]. In $40-50 \%$ of all affected children, teleangiectatic cutaneous vessels, fibrous-fatty tissue or scar formation can be observed as a residue of the lesion [4].

Although most hemangiomas have a self-limiting course, some may cause severe complications such as visual impairment, congestive (high cardiac output) heart failure and disfigurement [5]. Ulceration and infection can occur in up to $20 \%$ of cases [6], from which significant and permanent scarring of the skin may result, leading to cosmetic impairments that may have an impact on quality of life and future self-confidence of the patient.

The recently discovered effectiveness of propranolol in treating infantile hemangiomas has led the drug to be used as first line of therapy in the vast majority of medical centers. Although propranolol is well tolerated in most infants, there are concerns about the drug's potential cardiovascular, metabolic and behavioral side effects. Propranolol can cause hypotension, hypoglycemia, bradycardia, pulmonary hyper- reactivity and other transient symptoms like sleep disturbance, daytime drowsiness, digestive symptoms and poor weight gain [7].

\section{Methods}

We collected during 5 years 100 infantile hemangioma patients from three pediatrics department in Israel, of which all been treated with propranolol treatment course. Data extraction was based on patient hospital medical files and parent's reports during follow-up visits. The infants underwent comprehensive cardiovascular evaluation including 24 hour holter, echocardiography, blood pressure and heart rate before and during the treatment to evaluate the side effects and the regression of the lesions. We evaluate the effects of personal parameters like birth weight and gender, treatment characteristics like duration, maximal dose and initiation time on final treatment results and its side effects. 


\section{Results}

Our group treated 100 hemangioma patients with propranolol among them suspected side effects were observed in 17 patients (Table 1), of which only five required dose adjustment, complete, or temporary cessation of propranolol therapy. In three of the cases there existed doubts concerning the causative effect of propranolol with respect to the adverse effects observed.

Among the reported side effects, we emphasize the cases needed intervention.

\section{Case Reports}

\section{Case 1: Convulsive disorder (patient 1)}

A five months old female was referred to our pediatric cardiology clinic for the initiation of propranolol treatment for an upper lip hemangioma. The patient was born prematurely at 32 weeks, the second of two non-identical twins, with no signs of distress and a birth weight of 1,460 g. The hemangioma appeared a few weeks after birth. The infant underwent a full cardiovascular evaluation including cardiac echocardiography, ECG, blood pressure and heart rate measurements, which were all within normal ranges. A complete blood workup was performed from which no abnormalities could be readily observed. Propranolol treatment was initiated at an initial dose of $1 \mathrm{mg} / \mathrm{kg} /$ day with no adverse effects. Two weeks later, on the day the therapeutic dose of $2 \mathrm{mg} / \mathrm{kg} /$ day was obtained, the infant had her first convulsive episode. This episode followed a breath holding spell during extended crying and lasted approximately one minute with spontaneous resolution. One week later she had her second seizure, this time unaccompanied by crying. The next day her examination revealed no neurologic deficits except for general symmetric hypotonia. Additional tests revealed both blood glucose levels and EEG within normal range. The medical staff had strong doubts about the relation of the treatment to those episodes and the propranolol sustained. During the following two months she experienced a few more seizers, therefore, propranolol was discontinued despite the significant effect on the skin lesion. The infant was re-examined; a second EEG showed generalized paroxysmal fast activity at the initiation of sleep. With the working diagnosis of convulsion disorder the patient was started on valproic acid.

During those few months without propranolol treatment the seizures continued and the hemangioma underwent a substantial regression. Several attempts to continue the propranolol were made; during each attempt a substantial improvement of the hemangioma was observed, however when the first convulsion took place, the propranolol was discontinued by the infant's parents.

\section{Case 2: Bradycardia and asystole reports}

\section{Case 2A: (patient 2)}

A healthy ten month old female infant with two hemangiomas was referred to our pediatric cardiology clinic for initiating propranolol treatment. The lesions appeared a few weeks after birth. One of the lesions was on her scalp and the other was on the labium majus. Following a routine cardiovascular and blood evaluation which was normal, treatment protocol was initiated. The primary dose of $1 \mathrm{mg} / \mathrm{kg} /$ day oral propranolol was well tolerated in the clinic with no side effects. During the adjustment period to the treatment, the dose was increased to 2 $\mathrm{mg} / \mathrm{kg} / \mathrm{day}$, and mild side effects were reported by the parents including irritability and sleep disturbance. A 24 hour ECG Holter-monitoring detected episodes of bradycardia as a result of an increased vagal tone, with no asystole or complex arrhythmia presented. The propranolol dose was reduced to 1.5 $\mathrm{mg} / \mathrm{kg} /$ day. The treatment was well tolerated and one month later a successful attempt to increase the dose to $2 \mathrm{mg} / \mathrm{kg} /$ day ensued. This dose increase caused no side effects and a repeated Holter-monitoring showed a normal rate with no episodes of bradycardia.

\section{Case 2B: (patient 3)}

A three month old female was referred to our pediatric cardiology clinic due to a right lower lip hemangioma. The lesion appeared two weeks after birth. Following a baseline cardiovascular and blood evaluation which was normal a treatment protocol was initiated. The primary dose of 1 $\mathrm{mg} / \mathrm{kg} /$ day was well tolerated in the clinic with no side effects. Two weeks later when a therapeutic dose of $2 \mathrm{mg} / \mathrm{kg} /$ day was reached, a 24 hour ECG Holter-monitoring detected episodes of bradycardia and numerous premature ventricular beats. The propranolol dose was reduced to $1.5 \mathrm{mg} / \mathrm{kg} /$ day. The treatment was well tolerated, no side effects were detected and a repeated Holter-monitoring test showed a normal cardiac rate with no episodes of bradycardia.

\section{Case 2C: (patient 4)}

A three month old male infant was referred to our pediatric cardiology clinic for initiating propranolol treatment due to three hemangiomas. One hemangioma was located on his forehead, a second lesion on his left arm and a third lesion on the back of his neck. The lesions appeared immediately after birth. Following a routine cardiovascular and blood evaluation that was normal, a propranolol treatment protocol was initiated. The primary dose of $1 \mathrm{mg} / \mathrm{kg} /$ day was well tolerated in the clinic with no side effects. Two weeks later when the therapeutic dose of 2 $\mathrm{mg} / \mathrm{kg} /$ day was obtained, a 24 hour ECG Holter-monitoring detected one episode of asystole that lasted for 3.5 seconds.

\section{Case 3: Hypoglycemia (patient 5)}

A healthy four year old female patient started propranolol treatment due to three hemangiomas on her face and neck. The lesions appeared immediately after birth. Following a routine cardiovascular and blood evaluation that was normal, a propranolol treatment protocol was initiated. The primary dose of $0.5 \mathrm{mg} / \mathrm{kg} /$ day was well tolerated in the clinic with no side effects. This dose was titrated up to $2 \mathrm{mg} / \mathrm{kg} /$ day over a 72 hour period. The patient took no other medication and had no history of hypoglycemia. The patient received the dose without interruption for 3 months. On the day of the event she ate breakfast and had no fever, nausea, vomiting or diarrhea. A few 
hours after receiving her morning dose of propranolol she became pale, disoriented and unresponsive and was therefore, admitted for further evaluation. In the emergency room she was hemodynamically stable. Her glucose level was $12 \mathrm{mg} / \mathrm{dl}$ and no hypothermia was detected. She was rapidly revived with IV glucose and oral fluids. Propranolol treatment was consequentially terminated immediately.

On a follow-up examination after cessation of treatment the patient appeared well with no apparent sequelae or similar clinical episodes reported.

\section{Case 4: Poor weight gain (patient 6)}

A four months old male was referred to the pediatric cardiology clinic for commencement of propranolol treatment due to one hemangioma on his right medial eyelid. The lesions appeared a few weeks after birth. His birth weight was 2,630 g. Following a routine cardiovascular and blood evaluation that was normal, treatment initiation began for this baby, at a weight of $7,370 \mathrm{~g}\left(75^{\text {th }}\right.$ weight percentile). The primary dose of 1 $\mathrm{mg} / \mathrm{kg} /$ day was well tolerated in the clinic with no side effects. This dose was increased within a week up to $2 \mathrm{mg} / \mathrm{kg} /$ day. Upon follow-up examination we noticed that the patient was not gaining weight appropriate for his age, specifically, a weight gain of $90 \mathrm{~g}$ in two weeks was observed. The treatment continued with recommendation for more frequent meals. Two months later, the patient reached the $50^{\text {th }}$ percentile for weight. Caloric intake was increased to $1 \mathrm{ml}=1 \mathrm{kcal}$ (instead of $1 \mathrm{ml}=0.65 \mathrm{kcal}$ in breast milk/standard formula). In addition, the parents received strict instructions regarding the frequency of meals and night feeding. Further follow-up examinations showed sufficient weight gains as a result of the altered feeding program, and upon termination of the 15 month treatment schedule the patient was in the $50^{\text {th }}$ percentile for weight.

\section{Case 5: hyperactive airway: (patient 7)}

A healthy three month old male, born at term and weighing $3,360 \mathrm{~g}$, was noted to have a superolateral hemangioma inside his eye socket, proximal to the lacrimal fossa. The hemangioma caused mild exophthalmos with no eye damage or visual impairment. Another hemangioma was spotted on his chest.
The hemangiomas appeared in the first few weeks of life. The patient was started on propranolol at $1 \mathrm{mg} / \mathrm{kg} /$ day which was increased to $2 \mathrm{mg} / \mathrm{kg} /$ day after 14 days. He was fully immunized and had no known allergies. Family history is notable for his father having childhood asthma. The hemangiomas showed marked improvement during treatment. Five months later he achieved full regression of the lesions and treatment was terminated. Two months later the propranolol had to be resumed due to recurrence of the hemangiomas and treatment was well tolerated. One month after continuation of treatment, he suffered from an episode of wheezing and bronchiolitis was suspected. There were concerns among the staff whether the event might be associated with an upper respiratory tract viral infection. Propranolol was ceased by the parents for two months during which his wheezing disappeared. Due to rebound growth of the hemangioma propranolol was commenced with no further episodes of wheezing or need for a bronchodilator. The patient was treated with oral propranolol for almost a year until complete clearance of the hemangiomas.

\section{Case 6: Mild and transient side effects: (patients 8-17)}

Among the 17 children that reported having side effects from the treatment only five needed intervention. Seven of the children were reviewed in previous cases. The other patients with reported side effects suffered from mild and transient symptoms.

Overall, three patients had wheezing during an upper respiratory viral infection. No intervention was necessary and the respiratory disturbances disappeared upon resolution of the illness in each case.

Four patients were reported to have sleep disturbances and irritability. No intervention was necessary, parents were reassured that there was no cause for alarm and during subsequent follow-up visits the issue did not resurge. Three patients had significant weight loss (100 g in one week), parents were educated about frequent feeding and the infants gained weight by the next follow-up visit. One infant had transient hair lose.

Table 1: Reported side effects among 100 patients treated with propranolol for infantile hemangioma.

\begin{tabular}{|c|c|c|c|c|c|c|c|}
\hline \multicolumn{3}{|c|}{ Demographics } & \multicolumn{3}{|l|}{ Treatment } & \multirow[b]{2}{*}{ Side effect } & \multirow[b]{2}{*}{ Management } \\
\hline Patient & Birthweight (g) & Gender & Onset (days) & Duration (days) & Max dose (mg/kg/day) & & \\
\hline 1 & 1460 & Female & 141 & 305 & 2 & Convulsion & Cessation \\
\hline 2 & 2960 & Female & 314 & 295 & 2 & Bradycardia /Asystole & Dose adjusment \\
\hline 3 & 3500 & Female & 104 & Ongoing & 2 & Bradycardia /Asystole & Dose adjusment \\
\hline 4 & Unknown & Male & 92 & Ongoing & 2 & Bradycardia /Asystole & Dose adjusment \\
\hline 5 & 2584 & Female & 1481 & 95 & 2 & Hypoglycemia & Cessation \\
\hline 6 & 2630 & Male & 62 & 452 & 2 & Poor weight gain & UC \\
\hline 7 & 3360 & Male & 103 & 224 & 2 & Hyper reactive airway & UC \\
\hline
\end{tabular}




\begin{tabular}{|l|l|l|l|l|l|l|l|}
\hline 8 & 2000 & Female & 433 & 341 & 2 & Hyper reactive airway & UC \\
\hline 9 & 2740 & Female & 163 & 307 & 2 & Poor weight gain & UC \\
\hline 10 & 2185 & Male & 144 & 339 & 2 & Poor weight gain & UC \\
\hline 11 & 3700 & Female & 213 & 319 & 2 & Irritability & Hyper reactive airway \\
\hline 12 & 2380 & Female & 439 & 382 & UC & Hair lose \\
\hline 13 & 1260 & Female & 86 & 365 & 3 & Sleep disturbance & UC \\
\hline 14 & 2685 & Male & 183 & 170 & 2 & Sleep disturbance & UC \\
\hline 15 & 2100 & Female & 310 & 297 & 3 & Mild bradycardia & UC \\
\hline 16 & 3180 & Female & 57 & 339 & 295 & 2 & \\
\hline 17 & 1750 & Female & 115 & & &
\end{tabular}

\section{Discussion}

Propranolol has been used for the treatment of cardiac condition in children for over 3 years [8].

Propranolol has been effectively used worldwide since 2008 for the treatment of infantile hemangiomas and is well tolerated in most infants. However, due to systemic beta blockers having the potential to cause adverse effects and due to lack of experience with this indication of treatment, concerns still exist regarding the drug's potential cardiovascular, metabolic and behavioral side effects.

Propranolol can cause hypotension, hypoglycemia, bradycardia, pulmonary hyper- reactivity and other transient symptoms like sleep disturbance, daytime drowsiness, digestive symptoms and poor weight gain [9].

Most cases described in the literature regarding seizures during propranolol treatment presented with a seizure after the first dose in the initiation of treatment, while most of the events were hypoglycemia related.

Regarding our patient (Case 1), since propranolol was resumed in this patient without any seizure-like incidents, the probability of cause and effect between propranolol and seizures is highly unlikely.

As a beta blocker, propranolol decreases heart rate and in part, blood pressure as a result of negative chronotropic and inotropic effects on the heart. The effect of Propranolol on heart rate and blood pressure in children peak around two hours after an oral dose [10]. The consensus guidelines recommend heart rate and blood pressure measurement one and two hours after initiation of beta- blocker therapy and after a significant dose increase [9]. Routine cardiovascular monitoring that include ECG, blood pressure and heart rate measurement, is not recommended after at least one set of normal measurement once target dose has been achieved. Furthermore, there is no recommendation in the literature for 24 hour ECG Holtermonitoring. Although the effect of beta- blockers on blood pressure can be seen shortly after the first dose, the maximal effect is reached after 48 hours, corresponding with the accumulation of propranolol in plasma, after achieving a steady state [11]. Our data show that both hypotension and bradycardia/asystole during follow-up can be missed by following the recommendations of the consensus protocol.

Although the bradycardia events that were observed in this paper were considered not to be clinically relevant and had no therapeutic consequences, it is important for one to be aware that these events might occur among the healthy pediatric population. It is especially important to detect recurrent events as we are uncertain what influence these adverse effects have on cardiovascular, gross motor, or neurocognitive systems in the long term.

We believe that a single 24 hour ECG Holter- monitoring is necessary once the target dose has been achieved and further evaluation should include questioning about symptoms, providing data for therapy adjustment.

Propranolol for the treatment of infantile hemangioma is a relatively new indication [12]. Existing publications cited doses between 1 and $3 \mathrm{mg} / \mathrm{kg} /$ day. The effectiveness and safety of the treatment has been proven in this full range of doses [9]. According to the cases described above we believe in personalized medicine, adjustment of treatment protocol and dosage to each patient taking into consideration the improvement rate and side effects.

The association between propranolol and hypoglycemia in infants and children is well known and is noted in the product insert. However, this adverse effect is best recognized during the neonatal period with older infants and children considered to be at a low risk [13].

From the literature review of hypoglycemia associated with propranolol therapy in infants and children preformed in 2010 we can conclude that the vast majority of hypoglycemia events take place in the morning, after an overnight to 24 hour fast. Most of the reported patients who developed hypoglycemia were prescribed relatively low doses, suggesting that hypoglycemia associated with propranolol may not be dose dependent [9], never the less dosage of over $4 \mathrm{mg} / \mathrm{kg} / \mathrm{day}$ seems to put the patient at a higher risk [14]. 
Normal glucose homeostasis is thought to be impaired through inhibition of beta adrenergic mediated glycogenolysis, gluconeogenesis and lipolysis [9]. Children and infants seem to be at higher risk for this adverse effect because their glucose utilization rates are higher in the fasting state ( 3 fold higher in infants) [13]. This is partly attributed to an existing greater brain mass relative to their body weight [15]. In addition, glycogen stores are lower in infants and children compared with adults, thus leading to a reduced fasting ability [16]. As a result, a patient taking propranolol may be vulnerable to hypoglycemia during periods of prolonged fasting when counter regulatory mechanisms may fail.

Clinical manifestation of hypoglycemia in infants can vary widely. Mild hypoglycemia produces symptoms associated with counter- regulatory epinephrine action, including sweating, shakiness, tachycardia, anxiety and hunger [13]. With propranolol induced beta adrenergic blockade, early symptoms may be masked. Sweating is not typically blocked by beta blockers, therefor this may be a more reliable symptom for diagnosis [17]. More sever hypoglycemia produces symptoms of neuroglycopenia, inducing lethargy, stupor, seizures, apnea, poor feeding, loss of consciousness and hypothermia [13].

The most common predicting factors for hypoglycemia during the treatment are infants being younger than 1 year of age with limited glycogen storage, low birth weight, systemic illness and previous treatment with corticosteroids [18]. The latter is significant because adrenal suppression may result in loss of the counter regulatory cortisol response [19]. Our patient (Case 3) is a healthy 4 year old healthy girl, hence this is a very uncommon presentation which mandates further investigation for the cause of this event. Inborn errors of carbohydrate and fatty acid metabolism are uncommon and their association with hemangiomas is unknown.

Propranolol should be discontinued during intercurrent illness, especially in the setting of restricted oral intake. We also recommend regular food intake and receiving the medication every day at the same time, before meals and never overnight. Frequent feeds must be encouraged.

Hypoglycemia as a side effect of propranolol therapy needs to be viewed seriously; since it is known that repetitive and sustained hypoglycemia in infants may lead to permanent impairment of brain growth and function [15].

Manifestation of consistent poor weight gain as seen in our patient (case 4 ) is not very common and could be multifactorial. The patient weight should be monitored at every follow-up visit (especially for the escalation of the dose regimen) and frequent feeds must be encouraged.

It is widely accepted that propranolol and other non-selective beta- blockers are contraindicated in patients with asthma and Fatal adverse reactions due to propranolol had been described in adult patients asthma [20]. However, there are hardly any case reports of wheezing in children while taking propranolol without a history or an underlying asthma. Among the cases reported, all of them were adequately controlled with beta agonist and inhaled corticosteroids which suggests that propranolol may not be contraindicated if wheezing develops [21].

The mechanism by which propranolol may exacerbate wheezing is related to the receptors for bronchodilatation and bronchoconstriction. Bronchodilatation is mediated through catecholamine stimulation of beta 2 receptors in the lung. Non selective beta adrenergic blockers, such as propranolol, can cause bronchoconstriction [22]. This process may lead to an exaggerated bronchoconstriction response to usually nontoxic stimuli such as a viral illness or aeroallergens [21].

Wheezing is very common in infancy, with reported prevalence of $28.5 \%$ to $32 \%$ [23]. The case of bronchial hyper reactivity can be attributed simply to underlying viral bronchiolitis because severity of bronchospasm in this illness is highly variable in infants [24].

The cause of wheezing in our case (case 5) may have been multifactorial, and the authors cannot exclude the possibility of a viral induced wheeze.

\section{Conclusion}

During 2009-2015 one hundred infants with hemangiomas completed treatment with oral propranolol in our department. Suspected side effects were observed in 17 infants.

The vast majority of frequently reported side effects are transient and mild. It is imperative for caregivers and professionals to be educated in recognizing possible side effects of beta-blocker treatment in patients with infantile hemangiomas and act accordingly. This information should be given to the family at the first consultation.

Most side effects reported (12/17) were related to maternal (parental) coping with infant's behavior during treatment progress in the initial phase of propranolol therapy. Transient and mild adverse effects such as feeding problems, restlessness and sleeping pattern disturbances are very subjective. It is important to differentiate between complaints made due to misunderstanding stemming from parents performing online web searches to real, life threatening symptoms caused by medications given to children.

The absence of adverse effects during the monitored initial treatment does not preclude the possibility of developing serious adverse effect from propranolol later in the course of treatment. Educating parents is therefore, important for patient safety.

\section{References}

1. Kilcline C, Frieden IJ (2008) Infantile hemangiomas: How common are they? A systematic review of the medical literature. Pediatr Dermatol 25: 168-173.

2. Leaute-Labreze C, Dumas de la Roque E, Hubiche T, Boralevi F, Taieb A, et al. (2008) Propranolol for severe hemangiomas of infancy. New Engl J Med 358: 2649-2651.

3. Chang LC, Haggstrom AN, Drolet BA, Baselga E, Chamlin SL, et al. (2008) Growth characteristics of infantile hemangiomas: Implications for management. Pediatr 122: 360-367. 
4. Eivazi B, Ardelean M, Baumler W, Berlien HP, Cremer H, et al. Update on hemangiomas and vascular malformations of the head and neck. Eur Arch Otorhinolaryngolo 266: 187-197.

5. Dotan M, Lorber A (2013) Congestive heart failure with diffuse neonatal hemangiomatosis--case report and literature review. Acta paediatr 102: e232-238.

6. Chamlin SL, Haggstrom AN, Drolet BA, Baselga E , Frieden IJ, et al. (2007) Multicenter prospective study of ulcerated hemangiomas. J Pediatr 151: 684-689.

7. Sanchez-Carpintero I, Ruiz-Rodriguez R, Lopez-Gutierrez JC (2011) Propranolol in the treatment of infantile hemangioma: Clinical effectiveness, risks, and recommendations. Actas Dermosifiliogr 102: 766-779.

8. Dyme JL, Thampan A, Han EJ, Kotb ME, Shin HT, et al. (2012) Propranolol for infantile haemangiomas: Initiating treatment on an outpatient basis. Cardiol young 22: 424-429.

9. Drolet BA, Frommelt PC, Chamlin SL, Haggstrom A, Bauman NM, et al. (2013) Initiation and use of propranolol for infantile hemangioma: Report of a consensus conference. Pediatr 131: 128-140.

10. Cushing SL, Boucek RJ, Manning SC, Sidbury R, Perkins JA (2011) Initial experience with a multidisciplinary strategy for initiation of propranolol therapy for infantile hemangiomas. Otolaryngol Head Neck Surg 144: 78-84.

11. Fagan TC, Walle T, Corns-Hurwitz R, Conradi EC, Privitera P, et al. (1983) Time course of development of the antihypertensive effect of propranolol. Hypertension 5: 852-857.

12. Sans V, de la Roque ED, Berge J, Grenier N, Boralevi F, et al. (2009) Propranolol for severe infantile hemangiomas: Follow-up report. Pediatr 124: e423-431.

13. Holland KE, Frieden IJ, Frommelt PC (2010) Hypoglycemia in children taking propranolol for the treatment of infantile hemangioma. Arch dermatol 146: 775-778.

14. Chavez H, Ozolins D, Losek JD (1999) Hypoglycemia and propranolol in pediatric behavioral disorders. Pediatr 103: 1290-1292.
15. Duvanel CB, Fawer CL, Cotting J, Hohlfeld P, Matthieu JM (1999) Long-term effects of neonatal hypoglycemia on brain growth and psychomotor development in small-for-gestational-age preterm infants. J Pediatr 134: 492-498.

16. van Veen MR, van Hasselt PM, de Sain-van der Velden MG, Hofstede FC, Visser G, et al. (2011) Metabolic profiles in children during fasting. Pediatr 127: e1021-1027.

17. Lawley LP, Siegfried E, Todd JL (2009) Propranolol treatment for hemangioma of infancy: Risks and recommendations. Pediatr Dermatol 26: 610-614.

18. Liu LS, Sokoloff D, Antaya RJ (2013) Twenty-four-hour hospitalization for patients initiating systemic propranolol therapy for infantile hemangiomas--is it indicated? Pediatr Dermatol 30: 554-560.

19. Breur JM, de Graaf M, Breugem CC, Pasmans SG (2011) Hypoglycemia as a result of propranolol during treatment of infantile hemangioma: A case report. Pediatr Dermatol 28: 169-171.

20. Fallowfield JM, Marlow HF (1996) Propranolol is contraindicated in asthma. BMJ 313: 1486.

21. Shepherd D, Adams S, Wargon O, Jaffe A (2012) Childhood wheeze while taking propranolol for treatment of infantile hemangiomas. Pediatr Pulmonol 47: 713-715.

22. Patakas D, Argiropoulou V, Louridas G, Tsara V (1983) Betablockers in bronchial asthma: Effect of propranolol and pindolol on large and small airways. Thorax 38: 108-112.

23. Visser CA, Garcia-Marcos L, Eggink J, Brand PLP (2010) Prevalence and risk factors of wheeze in Dutch infants in their first year of life. Pediatr Pulmonol 45: 149-156.

24. King VJ, Viswanathan M, Bordley WC (2004) Pharmacologic treatment of bronchiolitis in infants and children: A systematic review. Arch Pediatr Adolesc Med 158: 127-137. 